\title{
A Randomized Placebo-Controlled Trial of Combination Therapy With Post-triple-antibiotic-therapy Fecal Microbiota Transplantation and Alginate for Ulcerative Colitis: Protocol
}

\section{OPEN ACCESS}

Edited by:

Javier Ochoa-Repáraz, Eastern Washington University,

United States

Reviewed by:

Taro Osada

Juntendo University Urayasu

Hospital, Japan

Shigeo Koido,

Jikei University Kashiwa

Hospital, Japan

Qianqian Li,

Nanjing Medical University, China

*Correspondence:

Dai Ishikawa

dai@juntendo.ac.jp

orcid.org/0000-0002-1130-2210

Specialty section:

This article was submitted to

Gastroenterology,

a section of the journal

Frontiers in Medicine

Received: 18 September 2021

Accepted: 19 January 2022

Published: 22 February 2022

Citation:

Ishikawa D, Zhang X, Nomura $K$,

Seki N, Haraikawa M, Haga K,

Shibuya T, Kim Y-G and Nagahara A

(2022) A Randomized

Placebo-Controlled Trial of

Combination Therapy With

Post-triple-antibiotic-therapy Fecal

Microbiota Transplantation and

Alginate for Ulcerative Colitis:

Protocol. Front. Med. 9:779205.

doi: 10.3389/fmed.2022.779205
Dai Ishikawa ${ }^{1,2 *}$, Xiaochen Zhang ${ }^{1}$, Kei Nomura ${ }^{1}$, Natsumi Seki $^{3}$, Mayuko Haraikawa ${ }^{1}$, Keiichi Haga ${ }^{1}$, Tomoyoshi Shibuya ${ }^{1}$, Yun-Gi Kim ${ }^{3}$ and Akihito Nagahara ${ }^{1,2}$

${ }^{1}$ Department of Gastroenterology, Juntendo University School of Medicine, Tokyo, Japan, ${ }^{2}$ Department of Intestinal Microbiota Therapy, Juntendo University School of Medicine, Tokyo, Japan, ${ }^{3}$ Research Center for Drug Discovery, Faculty of Pharmacy, Graduate School of Pharmaceutical Sciences, Keio University, Tokyo, Japan

Background: Fecal microbiota transplantation (FMT) has been widely performed for ulcerative colitis (UC) treatment at the clinical trial stage. Previous reports have used multiple FMT methods to enhance the colonization of healthy donor microbiota in the recipient's intestines. FMT following triple antibiotic therapy with amoxicillin, fosfomycin, and metronidazole (A-FMT) is not only effective but also requires only one FMT, which improves dysbiosis caused by reduced Bacteroidetes diversity in patients with UC. Alginate and its derivatives have the potential to induce the growth of intestinal bacteria including Bacteroides members and produce short-chain fatty acids (SCFAs), which are beneficial in regulating overactive autoimmunity. Our trial aims to investigate whether post-intervention with alginate, which can improve the intestinal environment, will enhance the therapeutic effect of A-FMT in UC and increase the long-term remission rate.

Methods and Analysis: This trial is a double-blinded, randomized, placebo-controlled, parallel assignment trial. Patients with UC and fecal donation candidates will undergo strict screening before being involved in the trial. Eligible patients are randomly divided into two groups: one group will drink one bottle of alginate twice a day for 8 consecutive weeks after A-FMT, while the other group will take a placebo instead of the alginate drink. The primary endpoints are the changes in the Total Mayo Score at 8 weeks after study initiation and A-FMT from baseline. The secondary endpoint is the comparison of clinical features, microbiota, and metabolomic analysis before and after 8 weeks of study food intake. Changes at 6, 12, 18, and 24 months after A-FMT will be assessed. Finally, a subpopulation analysis of the relationship between patients and donors is an exploratory endpoint.

Discussion: The FMT post-treatment used in this study is an oral alginate drink that is easily accepted by patients. If the regimen achieves the desired results, it 
can further improve the A-FMT regimen and provide evidence for clinical practice guidelines for UC.

\author{
Clinical Trial Registration: https://jrct.niph.go.jp/latest-detail/jRCTs031200103, \\ identifier: jRCTs031200103.
}

Keywords: ulcerative colitis, alginate, fecal microbiota transplantation (FMT), double-blind randomized controlled trial, antibiotic-FMT, placebo-controlled clinical study

\section{INTRODUCTION}

Although advances in ulcerative colitis (UC) drug treatment regimens have been made, $20-25 \%$ of patients choose a surgical intervention due to resistance or intolerance to drug treatment, which can cause pain and inconvenience to patients (1). To date, the pathogenesis of UC is not completely understood, and is only known to be the result of autoimmune processes and genetic and environmental factors; however, patients with UC commonly show decreased diversity and richness in the intestinal microbiota, resulting in dysbiosis $(2,3)$. Dysbiosis is thought to contribute to the development of aberrant immunological responses in inflammatory bowel disease (IBD) (4).

Fecal microbiota transplantation (FMT) is a minimally invasive therapeutic approach that involves transplanting intestinal microbiota from healthy donors to restore the normal intestinal microbiota functions in patients with disease-related dysbiosis. The implementation of FMT to treat UC is rapidly attracting the attention of the public $(5,6)$. Randomized clinical trials have assessed the use of multiple FMT operations for enhancing the colonization of the intestinal microbiota from healthy donors $(7-10)$. The clinical remission rate of patients receiving FMT reached an average of $42.1 \%$ (11). However, the methods outlined in the above reports are difficult to manage and implement in actual clinical practice.

FMT following triple antibiotic therapy with amoxicillin, fosfomycin, and metronidazole (A-FMT), a method used by our team, is simple to operate because it only requires one FMT via colonoscopy followed by administration of antibiotics for 2 weeks. The combination antibiotic therapy consisting of amoxicillin, fosfomycin, and metronidazole (AFM) was modified for amoxicillin, tetracycline and metronidazole (ATM) therapy to reduce the adverse events caused by tetracycline. The efficacy of these combination antibiotic therapies (AFM, ATM) in treating patients with UC was previously reported $(12,13)$. We employed AFM as antibiotic pre-treatment to enhance reprogramming of the host intestinal microbiota by increasing the donor microbe colonization (14) and to achieve synergistic effects with FMT. We previously reported that A-FMT contributed to the recovery of the Bacteroidetes composition, which is associated with clinical responses and UC severity (15). It has also been observed that Bacteroidetes species components in clinical responders treated with A-FMT remarkably resembled those of their donors (16).

Abbreviations: UC, ulcerative colitis; IBD, inflammatory bowel disease; FMT, fecal microbiota transplantation; A-FMT, FMT following triple antibiotic therapy AFM (amoxicillin, fosfomycin, and metronidazole); FCSA, short-chain fatty acids; CDI, Clostridium difficile infection; HAMD, Hamilton Depression Scale; NPI, Neuropsychiatric Inventory; PEG, polyethylene glycol.
These results indicate that A-FMT can effectively transplant the Bacteroidetes cells which is lost as UC activity progress (17) in fecal specimens from the donor to the intestinal environment of the recipient, which is parallel to the clinical improvement of UC. Furthermore, the high average clinical remission rate $(43.8 \%)$ was observed in patients at four weeks after administering A-FMT comparing to previous reports with conventional FMT methods $(42.1 \%)(15,18)$. Although A-FMT exhibited obvious advantages compared to AFM monotherapy in our long-term clinical study, the remission rate gradually declined to $18.2 \%$ within 24 months (18). Wei et al. reported that intervention with pectin, a soluble dietary fiber extracted from apples, after FMT can preserve the diversity of the intestinal microbiota of patients with UC, providing results similar to those of donors (19). We speculate that FMT combined with post-interventions to maintain the diversity of the intestinal microbiota may improve the efficacy of FMT in UC treatment.

Alginic acid is a soluble dietary fiber polysaccharide that is widely distributed in the cell walls of brown algae in the form of alginate (20). Previous reports have found that alginate oligosaccharides have various activities, such as anti-inflammatory activity (21), antifungal activity (22) and immunomodulatory activity (23). Besides, alginate not only has a protective function on mucous membranes of the upper gastrointestinal tract (24), but also ameliorated the symptoms of experimental colitis and inflammatory responses (25). Mirshafiey et al. have also verified that alginate can be used as a potential treatment option for UC in both acute and chronic phase by rat models $(26,27)$. Furthermore, alginate and its derivatives can improve the growth of intestinal bacteria and can be fermented by human gut bacteria to produce short-chain fatty acids (SCFAs), which are beneficial to the intestine (28-30). A metaanalysis showed that clinical improvements in IBD are associated with the abundance of fecal microbiota and the enrichment of SCFA-producing anaerobes (31). Therefore, the aims of our study are to determine whether post-intervention with alginate can increase the diversity of microbiota to enhance the therapeutic effect of A-FMT in UC and delay the loss of microbiota diversity to improve the long-term remission rate. To our knowledge, this is the first randomized controlled study of the relationship between alginate and UC therapy.

\section{METHODS AND ANALYSIS}

\section{Study Design and Patients}

This double-blinded randomized, placebo-controlled, parallel assignment trial will be conducted at Juntendo University Hospital (Tokyo, Japan). The study will be conducted until 
TABLE 1 | Exclusion criteria for patients with UC.

Informed consent not provided

Infectious enterocolitis

Receiving local therapy

Serious disease, such as liver disease, kidney disease, heart disease,

or other serious complications

Autoimmune disease

Pregnant women and all cases with the possibility of pregnancy

Allergic diseases

Antibiotic therapy in the past 3 months

Any other cases judged inappropriate by the responsible researcher

UC, Ulcerative colitis.

December 31, 2024 and is expected to involve 60 patients with UC. Diagnosis of UC will be established based on standard clinical, endoscopic, and histological findings (32) and both hospitalized patients and outpatients will be included. To be eligible, patients must be diagnosed with active UC and meet the requirements of obtaining a Total Mayo Score of 3-10 (7, 9, 10) and a Sum Endoscopic Mayo Score of 2 or above. All patients will be over 20 years old and competent enough to provide informed consent. There are no restrictions on gender.

To ensure the safety of patients, rigorous screening of patients will be performed. If the patient has serious illness, pregnant women, or has received local therapy, etc. will not be included in our experiment. The detailed exclusion criteria for this study are listed in Table $\mathbf{1 .}$

\section{Donors}

To understand the health status of the donors and reduce additional costs, we will conduct a preliminary medical inquiry and physical examination of the donor candidates. As dysbiosis is correlated with neuropsychiatric disorders including depression and autism (gut-brain interaction) $(33,34)$, the Hamilton Depression Scale (HAMD) and Neuropsychiatric Inventory (NPI) will be used to assess psychiatric symptoms (Table 2A). There are no restrictions on gender. In addition, to minimize the risk of infection transmission, the donor candidates who have passed the preliminary screening will undergo fecal and blood tests. Donor candidates who test positive for any of the items in Tables 2B, 2C will be excluded. Candidates who pass the above rigorous screening will become eligible donors. Finally, eligible donors will confirm whether they have diarrhea, fever, or other uncomfortable symptoms when submitting stool, as well as whether any cohabitants have diarrhea symptoms. The donor candidates recommended by the patients themselves will also need to pass all the screening processes mentioned above. Approximately $150-200 \mathrm{~g}$ of fresh stool provided by the eligible donors will be dissolved in $500 \mathrm{ml}$ of sterile normal saline. The sample will be processed to filter out crude fiber, and 100\% glycerol will be added to make the final glycerol concentration $10 \%$. The sample will then be divided into $200-\mathrm{ml}$ bottles (Corning Co., Ltd., NY, USA). This procedure will be performed under an anaerobic environment by replacing air with nitrogen
TABLE 2A | Donor exclusion criteria.

Medical interview (exclusions)
Age $<18$ or $>70$
BMl $<18$ or $>25$ or metabolic syndrome
Informed consent not provided
International travel to area with high risk of traveler's diarrhea in the last 6
months
High-risk sex (unprotected sex outside of a monogamous relationship in
last 3 months, or men who have sex with men, sex for drugs or money)
Tattoo, body piercing, or acupuncture in the last 6 months
Needle stick accident in the last 6 months
Household members with active gastrointestinal infection
History of vaccination with a live attenuated virus in the last 3 months
Incarceration or a history of incarceration
Medical history (exclusions)
History of major gastrointestinal surgery
Family history of colorectal carcinoma
Active medical illness or symptoms
Antimicrobials (antibiotics, antivirals, antifungals), probiotics, or PPls in the
last 3 months
Taking any medications
Acute diarrhea in the last 3 months

Irritable bowel syndrome, chronic constipation, Chronic diarrhea Other intrinsic gastrointestinal illness: Inflammatory bowel disease, Colonic polyps, Colon cancer

Autoimmune disease

Atopic disease (including atopic dermatitis)

Chronic fatigue syndrome

Psychiatric symptoms (exclusion)

Any psychiatric disorder assessed by HAMD or NPI

BMI, body mass index; PPIs, proton pump inhibitors; HAMD, Hamilton Depression Scale; $\mathrm{NPI}$, Neuropsychiatric inventor.

gas in a glove box (SANPLATEC Co., Ltd., Osaka, Japan). The fecal suspension will be cryopreserved at $-60^{\circ} \mathrm{C}$ in advance.

\section{Interventional Methods}

First, eligible patients with UC will receive a combination antibiotic regimen called AFM comprising oral amoxicillin (1,500 mg/day), fosfomycin (3,000 mg/day), and metronidazole (750 mg/day). AFM will be administered to patients for 2 weeks until 2 days before the FMT (15). The fecal suspension $(200 \mathrm{ml})$ will be thawed at $37^{\circ} \mathrm{C}$ using a water bath shaker on the day of the FMT operation. After bowel lavage using a standard polyethylene glycol (PEG) solution (Moviprep; EA Pharma, Tokyo, Japan), the patients will undergo total colonoscopy. A total of $200 \mathrm{~mL}$ of fecal suspension will be transferred to the patient's cecum. After the procedure, patients will receive scopolamine $(10 \mathrm{mg})$ to slow intestinal transit and maintain a right lateral position. If the patient's drug dose for UC treatment has been stable for at least 12 weeks before the preregistration, the patient will be permitted to continue ongoing treatment. However, changes in the dosage of drugs or initiation of new treatments will not be allowed during the intervention. After the A-FMT regimen described above, the patients will 
TABLE 2B | Donor screening criteria: blood test.

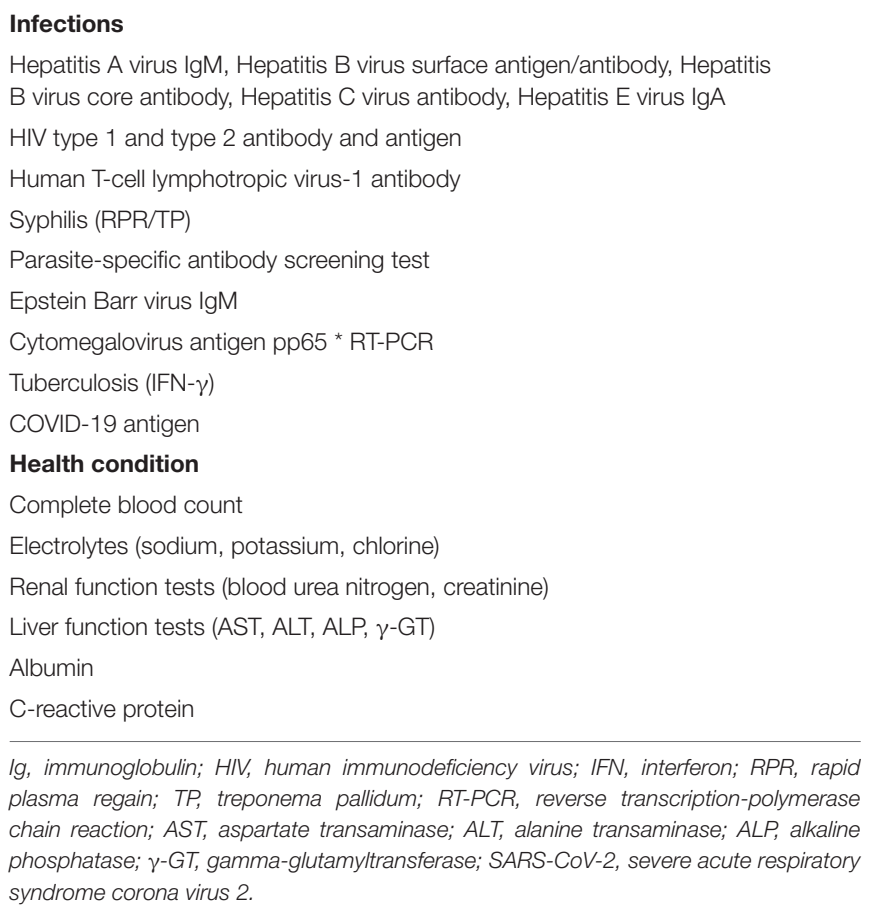

Ig, immunoglobulin; HIV, human immunodeficiency virus; IFN, interferon; RPR, rapid plasma regain; TP, treponema pallidum; RT-PCR, reverse transcription-polymerase chain reaction; $A S T$, aspartate transaminase; $A L T$, alanine transaminase; $A L P$, alkaline phosphatase; $\gamma$-GT, gamma-glutamyltransferase; SARS-CoV-2, severe acute respiratory syndrome corona virus 2.

TABLE 2C | Donor screening criteria: fecal test.

Fecal occult blood testing
Parasites, ova, cysts
Cryptosporidium
Giardia
Norovirus
Rotavirus
General bacterial culture for common enteric pathogens
Salmonella
Shigella
Yersinia
Campylobacter
Escherichia coli
Diarrheagenic Escherichia coli
Enterohemorrhagic Escherichia coli
Escherichia coli verotoxin

Clostridium difficile toxin/ Clostridium difficile-specific GDH

GDH, glutamate dehydrogenase.

be randomized into two groups for a double-blind FMT posttreatment. One group will consume one bottle of alginate drink twice a day for 8 consecutive weeks, while the other group will take a placebo. The alginate drink is a mixed tea drink provided by Kaigen Pharma Co., Ltd. (Osaka, Japan) containing $4 \mathrm{~g}$ of sodium alginate per bottle $(150 \mathrm{~g})$ and has been proven to be safe (https://www.h-food.or.jp/dbadm/media/ 231cec6ad1478e2d429f733d93a7b272.pdf). The placebo uses the same tea drink with the sodium alginate removed and does not have a different appearance or taste. Patients can adjust their medication during post-observation after the study food intervention. To compare the effects of treatment, all patients will undergo symptom checking and colonoscopy and have blood and fecal samples tested at the times indicated in Table 3. Colonoscopy founding at screening, A-FMT and 8 weeks after study food intake should be taken video and judged by a third-part expert to confirm the scope and severity of UC lesions. Biopsy of inflammatory sites will be used to evaluate the histological score. Blood samples will be used to test routine blood biochemistry, and plasma components will be separated by centrifugal force and cryopreserved at $-60^{\circ} \mathrm{C}$. Fecal samples will be collected to determine the occult blood condition and calprotectin levels and analyze the intestinal microbiota and metabolites.

\section{Outcomes}

The primary endpoint is to compare the changes in the Total Mayo Score at 8 weeks after study initiation and A-FMT from baseline between alginate intake group and placebo intake group. The secondary endpoints are the comparison of the following items at 8 weeks after study initiation and A-FMT from baseline:

- The change in the Sum Endoscopic Mayo Score.

- Analysis of intestinal microbiota by $16 \mathrm{~S}$ rRNA gene amplicon sequencing and whole-genome sequencing.

- Metabolomic analysis: In addition to SCFAs in stool samples, the amount of lipids, free fatty acids, bile acids, and other clinical markers will also be measured by liquid chromatography tandem mass spectrometry (35).

We will also do same analysis as (1), (2), and (3) at 6, 12, 18, and 24 months after 8 weeks post-study initiation.

Subpopulation analysis of the relationship between patients and donors is considered an exploratory endpoint.

Clinical features will be judged by the Total Mayo Score, and the mucosal appearance of the Mayo Score will be evaluated by endoscopy. To evaluate the overall status of the intestine, endoscopic findings will be evaluated using the Sum Endoscopic Mayo Score, which refers to the sum of the scores of the seven segments (appendicular region, cecum, ascending colon, transverse colon, descending colon, sigmoid colon, and rectum). Intestinal microbial analysis will assess changes in composition and diversity. Metabolomic analysis will evaluate the metabolites of microbiota and elucidate the mechanism of action of alginate in the treatment of UC.

\section{Adverse Events}

Any adverse event caused by antibiotic (12), FMT therapy, study food intake, blood sampling, and endoscopy should be recorded in detail, including symptoms, time, duration, and follow-up until complete resolution or termination of treatment. If there is any serious adverse event that may lead to death, life-threatening, or intolerability, the study will be terminated and treatment will begin immediately. 
TABLE 3 | Protocol for sequential therapy of A-FMT with alginate drink or placebo.

\begin{tabular}{|c|c|c|c|c|c|c|c|}
\hline \multirow{3}{*}{ Item } & \multicolumn{2}{|c|}{ Screening stage } & \multicolumn{4}{|c|}{ Duration of administration } & \multirow{3}{*}{$\begin{array}{c}\text { Post- } \\
\text { observation } \\
\begin{array}{c}\text { 6, } 12,18 \text {, and } \\
24 \text { months } \\
\text { after }\end{array}\end{array}$} \\
\hline & \multirow{2}{*}{$\begin{array}{l}\text { Pre-registration } \\
\text { (within } 2 \text { months) }\end{array}$} & \multirow{2}{*}{$\begin{array}{l}\text { Screening (within a } \\
\text { month) }\end{array}$} & \multirow{2}{*}{\begin{tabular}{|c|} 
AFM \\
2 weeks
\end{tabular}} & \multirow[t]{2}{*}{ A-FMT } & \multicolumn{2}{|c|}{ Alginate drink or Placebo } & \\
\hline & & & & & 4 weeks after & 8 weeks after & \\
\hline Informed consent & $\bullet$ & & & & & & \\
\hline Check of patient background & $\bullet$ & & & & & & \\
\hline Administration of study food & & & & & $\bullet$ & $\bullet$ & \\
\hline Check of symptoms & $\bullet$ & $\bullet$ & & - & $\bullet$ & $\bullet$ & $\bullet$ \\
\hline Endoscopy & & - & & $\bullet$ & & $\bullet$ & \\
\hline Blood samples & & $\bullet$ & & $\bullet$ & & $\bullet$ & $\bullet$ \\
\hline Fecal samples & & $\bullet$ & & $\bullet$ & & $\bullet$ & $\bullet$ \\
\hline
\end{tabular}

A-FMT, FMT following triple antibiotic therapy AFM (amoxicillin, fosfomycin, and metronidazole).

TABLE 4 | The baseline characteristics of the study participants.

\begin{tabular}{lc}
\hline Items & All $(\boldsymbol{n}=\mathbf{3 3})$ \\
\hline Age & $40.5 \pm 12.0$ \\
Sex (M/F) & $22 / 11$ \\
Duration of disease (years) & $7.3 \pm 5.4$ \\
Disease location & \\
Proctitis & 4 \\
Left-sided colitis & 14 \\
Extensive colitis & 15 \\
Total Mayo score & $7.12 \pm 1.9$ \\
Mild: 3-5 & 8 \\
Moderate: 6-10 & 25 \\
Sum endoscopic mayo score & $6.5 \pm 4.2$ \\
Ongoing treatment & \\
5-ASA & 30 \\
Corticosteroid & 2 \\
Azathioprine & 4 \\
Vedolizumab & 1 \\
Anti-TNF & 3 \\
\hline
\end{tabular}

5-ASA, 5-aminosalicylic acid; Anti-TNF, anti-tumor necrosis factor.

\section{Progress and Estimated Study Period}

This trial began on August 25, 2020. The first study participant was enrolled on September 28, 2020. At present, 33 eligible patients have been included in our study on December 8, 2021. We summarized the baseline characteristics of the 33 patients (Table 4). If the number of participants continues to increase at this pace, this trial is expected to end in October 2022.

\section{Sample Size Estimation}

In our previous clinical trials (15), $\sim 40$ eligible patients with UC were collected in 1 year. Therefore, conservative estimates predict that 60 patients can be collected over 3 years. Eligible patients with UC will be randomized and assigned to two groups with a ratio of $1: 1$ in a double-blinded fashion. The estimated difference between each group in the Total Mayo Score as the primary endpoint from baseline is -2 , and the standard deviation of each group is estimated to be $\sim 2.5$. If a two-sample $t$-test is used to test the difference between groups, a power analysis predicts that statistical significance with a power of $\sim 86.1 \%$ can be reached.

\section{Statistical Analysis}

Relationships between Total Mayo Scores and Sum Endoscopic Mayo Scores will be assessed using a $t$-test. Furthermore, the correlation between the relative abundance of Bacteroidetes species using a Pearson's correlation coefficient will be evaluated. Differences will be considered significant at $P<0.05$.

\section{Randomization}

Eligible patients will be randomized at a ratio of 1:1 using an Interactive Web Response System (REDCap: Research Electronic Data CAPture). The simple randomization is carried out by the staff in charge of the clinical research and trial Center of the Juntendo University Hospital to ensure concealment of allocation. The medical staff, assistants and patients of our study team did not know the condition of random allocation until the experiment was completely finished.

\section{DISCUSSION}

FMT is a well-established treatment regimen for recurrent Clostridium difficile infection (CDI). Clinical practice guidelines clearly state that FMT can be used as a treatment option for CDI $(36,37)$. However, using FMT for UC treatment remains in the clinical trial stage. Since 2014, we have been committed to studying how to enhance the therapeutic effect and operational feasibility of FMT on UC in clinical trials. The proposed AFMT method has a significant effect on the treatment of UC in our previous studies. We hope to combine acceptable and convenient post-treatment methods based on A-FMT to further enhance the effect and prolong the remission period, including alginate supplements.

Alginate has the potential to increase the relative abundance of some beneficial Bacteroidetes members, resulting in an improved 
intestinal environment (38-40). Therefore, we hypothesize that the diversity of microbiota and the proportion of Bacteroidetes in the group treated with A-FMT combined with alginate will be higher than that in the placebo group. An increased number of Bacteroidetes species can suppress the inflammatory response through zwitterionic capsular polysaccharides, which are bacterial products that modulate $\mathrm{T}$ cells to secrete the antiinflammatory interleukin-10 (41). It has also been reported that alginate can be fermented by specific bacteria in feces, such as Bacteroides ovatus, Bacteroides xylanisolvens, and Bacteroides theaiotaomicron $(30,42)$, which can induce colonic regulatory $\mathrm{T}$ cells and promote anti-inflammatory effects $(28,43)$. Therefore, we believe that oral supplementation of alginate with exogenous sources can contribute to the intestinal anti-inflammatory response. Furthermore, we expect that the group treated with A-FMT combined with alginate will demonstrate better results in terms of clinical features and microbial analysis, and will retain its advantage over the placebo arm after 2 years' follow-up. The metabolites produced by microbiota can shape the colonic environment through a variety of activities, such as participating in signaling, immune system modulation, and antibiotic activity (44-46). However, it is not fully known how specific microbes and the small molecules they modulate interact to cause or control the inflammatory response. Therefore, in our study, a wide range of metabolomic analyses other than SCFAs will be included in the study.

Although this clinical study is limited to patients in single center, our method can also be applied to patients in other

\section{REFERENCES}

1. Kobayashi T, Siegmund B, Le Berre C, Wei SC, Ferrante M, Shen B, et al. Ulcerative colitis. Nat Rev Dis Primers. (2020) 6:74. doi: 10.1038/s41572-020-0205-x

2. Nemoto $H$, Kataoka $K$, Ishikawa $H$, Ikata $K$, Arimochi $H$, Iwasaki $\mathrm{T}$, et al. Reduced diversity and imbalance of fecal microbiota in patients with ulcerative colitis. Dig Dis Sci. (2012) 57:2955-64. doi: 10.1007/s10620-012-2236-y

3. Kostic AD, Xavier RJ, Gevers D. The microbiome in inflammatory bowel disease: current status and the future ahead. Gastroenterology. (2014) 146:1489-99. doi: 10.1053/j.gastro.2014.02.009

4. Cammarota G, Ianiro G, Cianci R, Bibbò S, Gasbarrini A, Currò D. The involvement of gut microbiota in inflammatory bowel disease pathogenesis: potential for therapy. Pharmacol Ther. (2015) 149:191212. doi: 10.1016/j.pharmthera.2014.12.006

5. Smits LP, Bouter KE, de Vos WM, Borody TJ, Nieuwdorp M. Therapeutic potential of fecal microbiota transplantation. Gastroenterology. (2013) 145:946-53. doi: 10.1053/j.gastro.2013.08.058

6. Wang JW, Kuo CH, Kuo FC, Wang YK, Hsu WH, Yu FJ, et al. Fecal microbiota transplantation: review and update. $J$ Formos Med Assoc. (2019) 118(Suppl. 1):S23-31. doi: 10.1016/j.jfma.2018. 08.011

7. Costello SP, Hughes PA, Waters O, Bryant RV, Vincent AD, Blatchford P, et al. Effect of fecal microbiota transplantation on 8-week remission in patients with ulcerative colitis: a randomized clinical trial. JAMA. (2019) 321:15664. doi: 10.1001/jama.2018.20046

8. Moayyedi P, Surette MG, Kim PT, Libertucci J, Wolfe M, Onischi C, et al. Fecal microbiota transplantation induces remission in patients with active ulcerative colitis in a randomized controlled trial. Gastroenterology. (2015) 149:102-9.e6. doi: 10.1053/j.gastro.2015.04.001 regions, then provide more evidence for the treatment of patients with UC.

\section{ETHICS STATEMENT}

The study protocol was approved by the Ethics Committee of the Juntendo Institutional Review Board, Juntendo University School of Medicine, and the Clinical Study Committee of Juntendo University Hospital (Approval Number J20-011). The patients/participants provided their written informed consent to participate in this study.

\section{AUTHOR CONTRIBUTIONS}

DI and Y-GK: conceptualization. DI: methodology, validation, funding acquisition, writing-review and editing, and project administration. DI and $\mathrm{KN}$ : formal analysis, data curation, and visualization. $\mathrm{KH}$ and TS: investigation. XZ and NS: writingoriginal draft preparation. AN: supervision. $\mathrm{MH}, \mathrm{KH}$, and TS: resources. All authors have read and agreed to the published version of the manuscript.

\section{FUNDING}

This research was funded by the joint research course with Kirin Holdings Co., Ltd. The funder was not involved in the study design, collection, analysis, interpretation of data, the writing of this article or the decision to submit it for publication.

9. Paramsothy S, Kamm MA, Kaakoush NO, Walsh AJ, van den Bogaerde J, Samuel D, et al. Multidonor intensive faecal microbiota transplantation for active ulcerative colitis: a randomised placebo-controlled trial. Lancet. (2017) 389:1218-28. doi: 10.1016/S0140-6736(17)30182-4

10. Rossen NG, Fuentes S, van der Spek MJ, Tijssen JG, Hartman JH, Duflou A, et al. Findings from a randomized controlled trial of fecal transplantation for patients with ulcerative colitis. Gastroenterology. (2015) 149:110-8.e4. doi: 10.1053/j.gastro.2015.03.045

11. Narula N, Kassam Z, Yuan Y, Colombel JF, Ponsioen C, Reinisch W, et al. Systematic review and meta-analysis: fecal microbiota transplantation for treatment of active ulcerative colitis. Inflamm Bowel Dis. (2017) 23:17029. doi: 10.1097/MIB.0000000000001228

12. Nishikawa $\mathrm{Y}$, Sato $\mathrm{N}$, Tsukinaga $\mathrm{S}$, Uchiyama $\mathrm{K}$, Koido $\mathrm{S}$, Ishikawa D, et al. Long-term outcomes of antibiotic combination therapy for ulcerative colitis. Ther Adv Chronic Dis. (2021) 12:20406223211028790. doi: 10.1177/20406223211028790

13. Ohkusa T, Kato K, Terao S, Chiba T, Mabe K, Murakami K, et al. Newly developed antibiotic combination therapy for ulcerative colitis: a double-blind placebo-controlled multicenter trial. Am J Gastroenterol. (2010) 105:18209. doi: 10.1038/ajg.2010.84

14. Ji SK, Yan H, Jiang T, Guo CY, Liu JJ, Dong SZ, et al. Preparing the gut with antibiotics enhances gut microbiota reprogramming efficiency by promoting xenomicrobiota colonization. Front Microbiol. (2017) 8:1208. doi: 10.3389/fmicb.2017.01208

15. Ishikawa D, Sasaki T, Osada T, Kuwahara-Arai K, Haga K, Shibuya T, et al. Changes in intestinal microbiota following combination therapy with fecal microbial transplantation and antibiotics for ulcerative colitis. Inflamm Bowel Dis. (2017) 23:116-25. doi: 10.1097/MIB.0000000000000975

16. Ishikawa D, Sasaki T, Takahashi M, Kuwahara-Arai K, Haga K, Ito S, et al. The microbial composition of Bacteroidetes species in ulcerative colitis is effectively improved by combination therapy with fecal microbiota 
transplantation and antibiotics. Inflamm Bowel Dis. (2018) 24:25908. doi: 10.1093/ibd/izy266

17. Nomura K, Ishikawa D, Okahara K, Ito S, Haga K, Takahashi M, et al. Bacteroidetes species are correlated with disease activity in ulcerative colitis. $J$ Clin Med. (2021) 10:1749. doi: 10.3390/jcm10081749

18. Okahara K, Ishikawa D, Nomura K, Ito S, Haga K, Takahashi M, et al. Matching between donors and ulcerative colitis patients is important for longterm maintenance after fecal microbiota transplantation. J Clin Med. (2020) 9:1650. doi: 10.3390/jcm9061650

19. Wei Y, Gong J, Zhu W, Tian H, Ding C, Gu L, et al. Pectin enhances the effect of fecal microbiota transplantation in ulcerative colitis by delaying the loss of diversity of gut flora. BMC Microbiol. (2016) 16:255. doi: 10.1186/s12866-016-0869-2

20. Guo X, Wang Y, Qin Y, Shen P, Peng Q. Structures, properties and application of alginic acid: a review. Int J Biol Macromol. (2020) 162:61828. doi: 10.1016/j.ijbiomac.2020.06.180

21. Zhou R, Shi X, Gao Y, Cai N, Jiang Z, Xu X. Anti-inflammatory activity of guluronate oligosaccharides obtained by oxidative degradation from alginate in lipopolysaccharide-activated murine macrophage RAW 2647 cells. J Agric Food Chem. (2015) 63:160-8. doi: 10.1021/jf503548a

22. Tøndervik A, Sletta H, Klinkenberg G, Emanuel C, Powell LC, Pritchard MF, et al. Alginate oligosaccharides inhibit fungal cell growth and potentiate the activity of antifungals against Candida and Aspergillus spp. PLoS ONE. (2014) 9:e112518. doi: 10.1371/journal.pone.0112518

23. Xu X, Wu X, Wang Q, Cai N, Zhang H, Jiang Z, et al. Immunomodulatory effects of alginate oligosaccharides on murine macrophage RAW2647 cells and their structure-activity relationships. J Agric Food Chem. (2014) 62:316876. doi: $10.1021 / \mathrm{jf} 405633 \mathrm{n}$

24. Daigo K, Wada Y, Yamada C, Yamaji M, Okuda S, Okada M, et al. Pharmacological studies of sodium alginate. 1 Protective effect of sodium alginate on mucous membranes of upper-gastrointestinal tract. Yakugaku Zasshi. (1981) 101:452-7. doi: 10.1248/yakushi1947.101.5_452

25. Yamamoto A, Itoh T, Nasu R, Nishida R. Effect of sodium alginate on dextran sulfate sodium- and 2,4,6-trinitrobenzene sulfonic acid-induced experimental colitis in mice. Pharmacology. (2013) 92:108-16. doi: 10.1159/000353192

26. Razavi A, Khodadadi A, Eslami MB, Eshraghi S, Mirshafiey A. Therapeutic effect of sodium alginate in experimental chronic ulcerative colitis. Iran $J$ Allergy Asthma Immunol. (2008) 7:13-8.

27. Mirshafiey A, Khodadadi A, Rehm BH, Khorramizadeh MR, Eslami MB, Razavi A. Sodium alginate as a novel therapeutic option in experimental colitis. Scand J Immunol. (2005) 61:31621. doi: 10.1111/j.1365-3083.2005.01571.x

28. Smith PM, Howitt MR, Panikov N, Michaud M, Gallini CA. Bohlooly-Y M, et al. The microbial metabolites, short-chain fatty acids, regulate colonic Treg cell homeostasis. Science. (2013) 341:569-73. doi: 10.1126/science.1241165

29. Singh N, Gurav A, Sivaprakasam S, Brady E, Padia R, Shi H, et al. Activation of Gpr109a, receptor for niacin and the commensal metabolite butyrate, suppresses colonic inflammation and carcinogenesis. Immunity. (2014) 40:128-39. doi: 10.1016/j.immuni.2013.12.007

30. Li M, Li G, Shang Q, Chen X, Liu W, Pi X, et al. In vitro fermentation of alginate and its derivatives by human gut microbiota. Anaerobe. (2016) 39:19-25. doi: 10.1016/j.anaerobe.2016.02.003

31. Mocanu V, Rajaruban S, Dang J, Kung JY, Deehan EC, Madsen KL. Repeated fecal microbial transplantations and antibiotic pre-treatment are linked to improved clinical response and remission in inflammatory bowel disease: a systematic review and pooled proportion meta-analysis. J Clin Med. (2021) 10:959. doi: 10.3390/jcm10050959

32. Yoshino T, Nakase H, Ueno S, Uza N, Inoue S, Mikami S, et al. Usefulness of quantitative real-time PCR assay for early detection of cytomegalovirus infection in patients with ulcerative colitis refractory to immunosuppressive therapies. Inflamm Bowel Dis. (2007) 13:1516-21. doi: 10.1002/ibd.20253

33. Sanada K, Nakajima S, Kurokawa S, Barceló-Soler A, Ikuse D, Hirata A, et al. Gut microbiota and major depressive disorder: a systematic review and meta-analysis. J Affect Disord. (2020) 266:1-13. doi: 10.1016/j.jad.2020.01.102

34. Kang DW, Adams JB, Coleman DM, Pollard EL, Maldonado J, McDonough-Means S, et al. Long-term benefit of microbiota transfer therapy on autism symptoms and gut microbiota. Sci Rep. (2019) 9:5821. doi: 10.1038/s41598-019-42183-0
35. Franzosa EA, Sirota-Madi A, Avila-Pacheco J, Fornelos N, Haiser HJ, Reinker S, et al. Gut microbiome structure and metabolic activity in inflammatory bowel disease. Nat Microbiol. (2019) 4:293-305. doi: 10.1038/s41564-018-0306-4

36. Cammarota G, Ianiro G, Tilg H, Rajilić-Stojanović M, Kump P, Satokari R, et al. European consensus conference on faecal microbiota transplantation in clinical practice. Gut. (2017) 66:569-80. doi: 10.1136/gutjnl-2016-313017

37. McDonald LC, Gerding DN, Johnson S, Bakken JS, Carroll KC, Coffin SE, et al. Clinical practice guidelines for Clostridium difficile infection in adults and children: 2017 update by the Infectious Diseases Society of America (IDSA) and Society for Healthcare Epidemiology of America (SHEA). Clin Infect Dis. (2018) 66:987-94. doi: 10.1093/cid/ciy149

38. Wang $\mathrm{Y}, \mathrm{Han} \mathrm{F}, \mathrm{Hu} \mathrm{B}, \mathrm{Li} \mathrm{J}, \mathrm{Yu} \mathrm{W}$. In vivo prebiotic properties of alginate oligosaccharides prepared through enzymatic hydrolysis of alginate. Nutr Res. (2006) 26:597-603. doi: 10.1016/j.nutres.2006.09.015

39. Ai C, Jiang P, Liu Y, Duan M, Sun X, Luo T, et al. The specific use of alginate from Laminaria japonica by Bacteroides species determined its modulation of the Bacteroides community. Food Funct. (2019) 10:430414. doi: $10.1039 / \mathrm{C} 9 \mathrm{FO} 00289 \mathrm{H}$

40. Wang Y, Li L, Ye C, Yuan J, Qin S. Alginate oligosaccharide improves lipid metabolism and inflammation by modulating gut microbiota in high-fat diet fed mice. Appl Microbiol Biotechnol. (2020) 104:354154. doi: $10.1007 / \mathrm{s} 00253-020-10449-7$

41. Neff CP, Rhodes ME, Arnolds KL, Collins CB, Donnelly J, Nusbacher $\mathrm{N}$, et al. Diverse intestinal bacteria contain putative zwitterionic capsular polysaccharides with anti-inflammatory properties. Cell Host Microbe. (2016) 20:535-47. doi: 10.1016/j.chom.2016.09.002

42. Salyers AA, Vercellotti JR, West SE, Wilkins TD. Fermentation of mucin and plant polysaccharides by strains of Bacteroides from the human colon. Appl Environ Microbiol. (1977) 33:319-22. doi: 10.1128/aem.33.2.319322.1977

43. Arpaia N, Campbell C, Fan X, Dikiy S, van der Veeken J. deRoos P, Liu H, et al. Metabolites produced by commensal bacteria promote peripheral regulatory T-cell generation. Nature. (2013) 504:451-5. doi: 10.1038/nature12726

44. Dorrestein PC, Mazmanian SK, Knight R. Finding the missing links among metabolites, microbes, and the host. Immunity. (2014) 40:82432. doi: $10.1016 /$ j.immuni.2014.05.015

45. McHardy IH, Goudarzi M, Tong M, Ruegger PM, Schwager E, Weger JR, et al. Integrative analysis of the microbiome and metabolome of the human intestinal mucosal surface reveals exquisite inter-relationships. Microbiome. (2013) 1:17. doi: 10.1186/2049-2618-1-17

46. Wu GD. Diet, the gut microbiome and the metabolome in IBD. Nestlé Nutr Inst Workshop Ser. (2014) 79:73-82. doi: 10.1159/0003 60686

Conflict of Interest: Kaigen Pharma Co., Ltd (Osaka, Japan) provided the alginate drink and placebo for this clinical study.

The authors declare that the research was conducted in the absence of any commercial or financial relationships that could be construed as a potential conflict of interest.

The reviewer TO declared a shared affiliation with several of the authors, $\mathrm{DI}, \mathrm{XZ}, \mathrm{KN}, \mathrm{MH}, \mathrm{KH}, \mathrm{TS}$, and $\mathrm{AN}$, to the handling editor at time of review.

Publisher's Note: All claims expressed in this article are solely those of the authors and do not necessarily represent those of their affiliated organizations, or those of the publisher, the editors and the reviewers. Any product that may be evaluated in this article, or claim that may be made by its manufacturer, is not guaranteed or endorsed by the publisher.

Copyright (c) 2022 Ishikawa, Zhang, Nomura, Seki, Haraikawa, Haga, Shibuya, Kim and Nagahara. This is an open-access article distributed under the terms of the Creative Commons Attribution License (CC BY). The use, distribution or reproduction in other forums is permitted, provided the original author(s) and the copyright owner(s) are credited and that the original publication in this journal is cited, in accordance with accepted academic practice. No use, distribution or reproduction is permitted which does not comply with these terms. 